\title{
A new reference standard for citation analysis in chemistry and related fields based on the sections of Chemical Abstracts
}

\author{
Christoph NeuHaus, ${ }^{a}$ HANS-Dieter Daniel ${ }^{\mathrm{a}, \mathrm{b}}$ \\ ${ }^{\text {a }}$ ETH Zurich, Professorship for Social Psychology and Research on Higher Education, \\ Zähringerstrasse 24, CH-8092 Zurich, Switzerland \\ ${ }^{\mathrm{b}}$ Evaluation Office, University of Zurich, Mühlegasse 21, CH-8001 Zurich, Switzerland
}

\begin{abstract}
Citation analysis for evaluative purposes requires reference standards, as publication activity and citation habits differ considerably among fields. Reference standards based on journal classification schemes are fraught with problems in the case of multidisciplinary and general journals and are limited with respect to their resolution of fields. To overcome these shortcomings of journal classification schemes, we propose a new reference standard for chemistry and related fields that is based on the sections of the Chemical Abstracts database. We determined the values of the reference standard for research articles published in 2000 in the biochemistry sections of Chemical Abstracts as an example. The results show that citation habits vary extensively not only between fields but also within fields. Overall, the sections of Chemical Abstracts seem to be a promising basis for reference standards in chemistry and related fields for four reasons: (1) The wider coverage of the pertinent literature, (2) the quality of indexing, (3) the assignment of papers published in multidisciplinary and general journals to their respective fields, and (4) the resolution of fields on a lower level (e.g. mammalian biochemistry) than in journal classification schemes (e.g. biochemistry \& molecular biology).
\end{abstract}

\section{Introduction}

Citation analysis has become established as a widely used method for the assessment of the research performance of universities, institutes, and research groups [MOED, 2005]. Citation counts are seen as quantitative measure of the resonance and impact of publications among the scientific community. Plain citation counts per se are,

Received December 28, 2007

Address for correspondence:

CHRISTOPH NEUHAUS

E-mail: neuhaus@gess.ethz.ch

0138-9130/US \$ 20.00

Copyright (C) 2008 Akadémiai Kiadó, Budapest

All rights reserved 
however, quite meaningless [Kostoff, 2002; SChUBERT \& BraUn, 1996]. Publication activity and citation habits differ too greatly from field to field to allow assessment of the research performance of an institute or research group on the basis of absolute numbers. The assessment of research performance is always relative to a frame of reference, in terms of which the plain citation counts are interpreted. The frame of reference gives a standard for average citation rates, against which the research performance of an institute or research group can be compared. Through this comparison with a reference standard, mere absolute numbers are placed in a larger context and take on meaning. Other terms used for reference standard are baseline and reference value.

The selection of an appropriate reference standard is a crucial point in research evaluation, because the assessment of research performance depends on the comparison of the average citation rate of an institute or research group to a reference standard. The comparison reveals the relative standing of an institute or research group to a reference standard. But evaluation of research is only possible by "comparing 'like' with 'like"" [MARTIN \& IRVINE, 1983, P. 61]. Applying inappropriate reference standards may result in invalid conclusions about the research performance of an institute or research group [GLÄNZEL \& AL., 1999]. It therefore makes sense to examine the appropriateness of a reference standard as the case arises, especially when bibliometric analysis is used to inform decisions, such as decisions on the allocation of research funds within an institution.

According to SCHUBERT \& BRAUN [1996], there are basically three approaches to setting reference standards for the comparative assessment of research performance. Reference standards may be established on the basis of (a) fields of research, (b) journals, or (c) related records. In research evaluation reference standards on the basis of fields have proved useful [AKSNES, 2005; VAN LEEUWEN \& AL., 2003; VAN RAAN, 2004]. Reference standards on the basis of fields originate from VINKLER [1986] who introduced the Relative Subfield Citedness (RW). This reference standard is defined as the average Impact Factor of the journals dedicated to the respective field(s). Nowadays, a widely used reference standard is the mean Field Citation Score (FCSm) developed by the Centre for Science and Technology Studies (CWTS) at Leiden University [VAN LEEUWEN \& AL., 2003; VAN RAAN, 2004]. This indicator represents the average citation rate of papers published in the field(s) in which the institute or research group is active. The definition of fields is based on a classification of journals into subject categories originally developed by the Institute for Scientific Information (now owned by Thomson Reuters), and it is probably the most frequently used journal classification scheme in bibliometric analyses. ${ }^{1}$ Journals are classified as a whole in one

${ }^{1}$ Thomson Scientific uses various journal classification schemes in its products Current Contents, Essential Science Indicators, Journal Citation Reports, and Web of Science; further journal classification schemes have been developed by ipIQ, formerly CHI Research [HAMILTON, 2003; NOMA, 1986], Katholieke Universiteit Leuven, the Information Science and Scientometrics Research Unit of the Hungarian Academy of Sciences [GlänZel \& SChUbert, 2003], and Science and Technology Policy Research Unit of the University of Sussex [KATZ \& HiCKS, 1995]. 
or several subject categories. Individual papers are assigned indirectly to fields by means of the journals in which they appear [GLÄNZEL \& AL., 1999]. The FCSm indicator takes into account the document type (research article, letter, note, or review) as well as the publication year. In the calculation of the reference standard, self-citations are excluded [VAN LEEUWEN \& AL., 2003; VAN RAAN, 2004].

The average number of citations per publication (CPP) of an institute or research group can then be compared with this reference standard. The ratio CPP/FCSm gauges the observed impact of an institute or research group against the expected impact by virtue of papers published worldwide in the same field(s). The field-normalized citation counts thus provide information as to whether the research performance of an institute or research group is above or below the international average. Based on the long standing experience of CWTS, values of less than 0.5 can be considered as far below, between 0.5 and 0.8 as below, between 0.8 and 1.2 as about the same as, between 1.2 and 1.5 as above, and higher than 1.5 as far above the average impact of the field(s) [VAN LEEUWEN \& AL., 2003; VAN RAAN, 2004].

In general, the classification of journals into subject categories as developed by Thomson Reuters proves to be of great value for research evaluation. The Centre for Science and Technology Studies (CWTS) at Leiden University, the Information Science and Scientometrics Research Unit (ISSRU) at Budapest, and Thomson Reuters itself use in their bibliometric analyses reference standards based on journal classification schemes. Its limitations become obvious in the case of multidisciplinary journals such as Nature, Science, and Proceedings of the National Academy of Sciences, and of general journals such as Angewandte Chemie and Journal of the American Chemical Society [GLÄNZEL \& AL., 1999]. The first group publishes papers in a wide range of fields and is therefore classified as multidisciplinary. Consequently, the impact of papers published in multidisciplinary journals is compared with a rather heterogeneous group of papers. Here there can be no comparison of 'like' with 'like.' Furthermore, the delimitation of fields by subject categories yields an incomplete picture of the output of a given field. Using the journal classification scheme, papers published in multidisciplinary and general journals are not assigned to a specific specialist field, and so with the delimitation of a given field, a considerable fraction of the relevant literature is not captured [RINIA \& AL., 1993]. Consequently, reference standards such as the FCSm indicator are based on only a fraction of papers effectively published in a field.

Another limitation concerns the resolution of the classification scheme [KOSTOFF, 2002; Schubert \& Braun, 1996]. The Essential Science Indicators of Thomson Reuters, as an outstanding example, provide reference standards solely for 22 broad fields of research (such as "biology \& biochemistry," "chemistry," and "physics"). Such broad subject categories aggregate fields with rather diverse citation habits and may not be an adequate reference standard for the assessment of research performance. 
In general, the delimitation of fields by a journal classification scheme is less precise than by a subject classification applied on a paper-by-paper basis [GLÄNZEL \& AL., 1999]. According to SCHUBERT \& BRAUN [1996], reference standards based on journal classification schemes are suited only at a macro level (such as nations or universities); for citation analysis at the meso or micro level "it is sometimes unavoidable to use a classification scheme concerning not only the journals but every single paper" (p. 313). In discipline-oriented databases such as Chemical Abstracts, MEDLINE, or INSPEC, fields and subfields can be identified by means of a structured subject classification scheme. Each paper is assigned individually to a field and subfield. RINIA \& AL. [1993] found considerable differences between publication sets obtained by a journal classification scheme and a subject classification scheme, both in the numbers of publications and in the contents of publication sets. Although new methods to identify fields were developed - such as co-journal, co-citation and co-word analysis, these methods are little used to determine reference standards. Clustering research articles on the basis of their abstracts, Kostoff \& MARTINEZ [2005] found that the average citation rates become increasingly stratified as the clusters become smaller and more focused. According to Kostoff and Martinez, this suggests that a "[...] meaningful 'discipline' citation average may not exist, and the mainstream large-scale mass production semi-automated citation analysis comparisons may provide questionable results“ (p. 61). They conclude, that cross-field comparisons ,[...] require the manually intensive approach of identifying those few research papers most closely related to the paper of interest, and normalizing on those papers" (p. 61).

Although interesting, from our point of view such an approach is not practicable in research evaluation of departments, institutes or research groups, which has to deal with hundreds if not thousands of papers. We propose a different method for setting reference standards for chemistry and related fields that is based on the sections of the Chemical Abstracts database. The alternative reference standard is transparent, reproducible and overcomes some limitations of the journal classification scheme of Thomson Reuters.

\section{Methods and results}

To overcome the limitations of journal classification schemes, we propose an alternative reference standard for chemistry and related fields that is based on the sections of the Chemical Abstracts database. The database, published by Chemical Abstracts Service, represents the world's most important compendia of published literature in chemistry and related fields such as biology and life sciences, engineering sciences, materials sciences, medical sciences, and physics. Chemical Abstracts covers publications from more than 10,000 journals (whereof more than 1,500 core journals are indexed cover-to-cover) as well as books, conference proceedings, dissertations, 
technical reports, preprints and patents of chemical, biochemical, and chemical engineering interest.

Chemical Abstracts is divided into 80 different main sections, which in turn are arranged into five broad headings [CHEMICAL ABSTRACTS SERVICE, 1999]:

- biochemistry,

- organic chemistry,

- macromolecular chemistry,

- $\quad$ applied chemistry and chemical engineering, and

- $\quad$ physical, inorganic, and analytical chemistry.

Within a section, papers are segmented into subsections. The section "general biochemistry", for instance, is divided into the subsections (1) reviews, (2) subcellular processes, (3) nucleic acids and their constituents, (4) proteins and their constituents, (5) carbohydrates, (6) lipids, (7) membranes, (8) other. Each single paper in Chemical Abstracts is assigned to only one section and subsection according to its main subject thrust and interest. For section placement, the prime emphasis as presented by the author(s) is determinded by a highly trained document analyst. In the vast majority of cases, papers can be unequivocally assigned to a specific section and subsection [CAS, 1997]. If subject matter is mixed, however, and hence appropriate to other sections, cross-references are established. Cross-references indicate that subject content as emphasized by the author(s) has some substantial relation to another subject area. Minor relation to another subject area are not considered, as almost every paper embodies to one degree or another a variety of subject matters that may be peripheral to the main thrust and interest.

In contrast to the journal classification schemes, in this procedure also papers that were published in multidisciplinary and general journals are assigned to a specific field. Furthermore, papers are placed in a hierarchical subject classification scheme consisting of headings, sections and subsections. Thereby reference standards may be established on different levels of research fields. Finally, the guidelines and policies of Chemical Abstracts Service that identify the subject content and the arrangement of papers in Chemical Abstracts are well documented [CAS, 1997]. The descriptions clarify the section titles which are purely descriptive and therefore subject to more than one interpretation based on the scientific backgrounds, points of view, and objectives of different readers. Each section description consists of statements of subject coverage, rules for placement of related or borderline subjects, recommendations for section cross-references and arrangement of papers by subsection.

To assess research performance, the average citation rate of papers published in the section(s) in which the institute or research group is active, the mean Field Citation Score based on the sections of Chemical Abstracts $\left(\mathrm{FCSm}_{\mathrm{CA}}\right)$, has to be determined. 
NEUHAUS \& DANIEL: A new reference standard for citation analysis in chemistry

The average number of citations per publication (CPP) of an institute or research group can then be normalized with the $\mathrm{FCSm}_{\mathrm{CA}}$ indicator. Analogously to the CPP/FCSm indicator, the section-normalized citation counts provides information as to whether the research performance of an institute or research group is above or below the international average.

Taking the journal Angewandte Chemie as an example, we illustrate the limitations of the Thomson Reuters journal classification scheme in the case of general journals. We retrieved all communications (that is, research articles) published in the journal Angewandte Chemie in the year $2000(\mathrm{~N}=880)$ from the CAplus (Chemical Abstracts) database hosted by STN International, and analyzed their section assignments. In CAplus, the section and subsection is available in the classification code (/CC) search field; cross-references are provided in a separate search field (/SX). Focusing on the prime emphasis of subject content, we did not consider cross-references for this analysis.

In Angewandte Chemie in 2000, research results were reported most frequently from sections 78 (inorganic chemicals and reactions, 18.1\%), 29 (organometallic and organometalloidal compounds, 12.4\%), and 22 (physical organic chemistry, 7.3\%). More than half of the papers come under the heading organic chemistry; a further $30 \%$ of the papers can be assigned to physical, inorganic, and analytical chemistry and $11 \%$ of the papers to biochemistry. In the journal classification scheme of Thomson Reuters, however, Angewandte Chemie is classified as a whole as "chemistry, multidisciplinary." On average, research articles published in this subject category are cited 10.86 times in the period 2000-2004 (see Table 1). Research articles published in the subject category "organic chemistry," in contrast, are cited "only" 8.97 times. In the case of Angewandte Chemie, papers that according to Chemical Abstracts are to be assigned to organic chemistry are therefore compared with a reference standard that is too high by $21 \%$. The impact of the articles is assessed in a frame of reference that does not satisfy the demand that 'like' be compared with 'like.' When evaluating research performance, choosing an unsuitable reference standard can lead to invalid conclusions, as the following example shows: If an average citation rate of $\mathrm{CPP}=11.00$ for an organic chemistry paper is compared with the reference standard for the subject category "chemistry, multidisciplinary," the resulting impact is CPP/FCSm $=11.00 / 10.86=1.01$ - that is, the research performance is about equal to the international standard in the field (cf. [VAN LEEUWEN \& AL., 2003; VAN RAAN, 2004]). But if the average citation rate is compared to the reference standard for the subject category "chemistry, organic," the resulting impact is $\mathrm{CPP} / \mathrm{FCSm}=11.00 / 8.97=1.23$ - that is, it is higher than the international standard in the field. This means that the assessment of research performance depends decisively on the chosen reference standard. 
NEUHAUS \& DANIEL: A new reference standard for citation analysis in chemistry

Table 1. Bibliometric indicators for 10 selected subject categories of the Journal Citation Reports

\begin{tabular}{lrrr}
\hline Subject category & \multicolumn{1}{c}{ P } & \multicolumn{1}{c}{ C } & \multicolumn{1}{c}{ CPP } \\
\hline Biochemical research methods & 7,310 & 74,635 & 10.21 \\
Biochemistry \& molecular biology & 49,206 & 911,319 & 18.52 \\
Chemistry, analytical & 12,999 & 111,017 & 8.54 \\
Chemistry, applied & 7,013 & 42,405 & 6.05 \\
Chemistry, inorganic \& nuclear & 8,946 & 70,899 & 7.93 \\
Chemistry, medicinal & 5,243 & 44,063 & 8.40 \\
Chemistry, multidisciplinary & 18,995 & 206,290 & 10.86 \\
Chemistry, organic & 15,348 & 137,716 & 8.97 \\
Chemistry, physical & 21,780 & 197,198 & 9.05 \\
Physics, atomic, molecular \& chemical & 10,834 & 100,771 & 9.30 \\
\hline
\end{tabular}

Note. $\mathrm{P}=$ number of research articles published in $2000, \mathrm{C}=$ number of citations during the period 2000-2004 (including self-citations), $\mathrm{CPP}=$ average number of citations per research article. The bibliometric indicators are based on the CD-ROM version of the SCI Edition of the Journal Citation Reports. In 2000 the Journal Citation Reports used 169 subject categories.

Table 2. Bibliometric indicators for the biochemistry sections of Chemical Abstracts

\begin{tabular}{|c|c|c|c|c|}
\hline \multicolumn{2}{|c|}{ Section } & \multirow{2}{*}{$\begin{array}{c}\mathrm{P} \\
27,377\end{array}$} & \multirow{2}{*}{$\frac{C}{276,178}$} & \multirow{2}{*}{$\begin{array}{r}\text { CPP } \\
10.09\end{array}$} \\
\hline 1. & Pharmacology & & & \\
\hline 2. & Mammalian hormones & 16,355 & 215,604 & 13.18 \\
\hline 3. & Biochemical genetics & 17,602 & 258,331 & 14.68 \\
\hline 4. & Toxicology & 10,184 & 68,569 & 6.73 \\
\hline 5. & Agrochemical bioregulators & 2,947 & 6,975 & 2.37 \\
\hline 6. & General biochemistry & 10,058 & 197,886 & 19.67 \\
\hline 7. & Enzymes & 9,573 & 129,583 & 13.54 \\
\hline 8. & Radiation biochemistry & 3,202 & 24,237 & 7.57 \\
\hline 9. & Biochemical methods & 8,320 & 76,694 & 9.22 \\
\hline 10. & Microbial, algal, and fungal biochemistry & 9,894 & 111,759 & 11.30 \\
\hline 11. & Plant biochemistry & 9,091 & 66,749 & 7.34 \\
\hline 12. & Nonmammalian biochemistry & 6,110 & 66,601 & 10.90 \\
\hline 13. & Mammalian biochemistry & 12,608 & 223,535 & 17.73 \\
\hline 14. & Mammalian pathological biochemistry & 22,941 & 312,522 & 13.62 \\
\hline 15. & Immunochemistry & 17,328 & 270,462 & 15.61 \\
\hline 16. & Fermentation and bioindustrial biochemistry & 2,675 & 10,510 & 3.93 \\
\hline 17. & Food and feed chemistry & 9,344 & 34,509 & 3.69 \\
\hline 18. & Animal nutrition & 4,994 & 34,637 & 6.94 \\
\hline 19. & Fertilizers, soils, and plant nutrition & 4,155 & 11,813 & 2.84 \\
\hline 20. & History, education, and documentation & 2,554 & 10,406 & 4.07 \\
\hline
\end{tabular}

Note. $\mathrm{P}=$ number of research articles published in 2000, $\mathrm{C}=$ number of citations during the period 2000-2004 (including self-citations), $\mathrm{CPP}=$ average number of citations per research article.

The example of the journal Angewandte Chemie shows that general journals publish papers in a wide range of fields, but journal classification schemes fail to assess this topical heterogeneity as they assign individual papers indirectly to fields by means of the journals in which they appear.

For chemistry and related fields the proposed alternative reference standard is based on the sections of the Chemical Abstracts database. To determine the values of the reference standard we again used the CAplus database hosted by STN International. In 
the CAplus file, cited references are included for journal articles, conference proceedings, and basic patents from selected patent offices from 1997 to the present [WhitLEy, 2002; NeuHAUS \& DANIEL, 2008]. The online host STN International offers an unparalleled combination of features for searching and analyzing cited references, thus providing numerous possibilities for performing citation analysis [CHEMICAL ABStracts Service, 2005; MARX \& AL, 2001; Neuhaus \& DANIEL, 2008; RIDLEY, 2001]. As an example, we determined the values of the reference standard for the 20 sections arranged into the biochemistry heading. We retrieved all research articles published in 2000 in the biochemistry sections and searched their citations (including self-citations) received over a 5-year period in CAplus. Because CAplus does not provide a distinct document type for research articles, the dataset was isolated by excluding papers with non-relevant document types, such as conference proceedings, letters, and reviews. Again we did not consider cross-references, i.e. the main emphasis of subject content is the determining factor for the reference standard.

In 2000 , a total of 207,312 research articles were published in the biochemistry sections. They accumulated 2,407,560 citations during the period 2000-2004 and thus were cited 11.61 times on average. Table 2 shows the number of research articles and citations as well as the average number of citations per research article for the biochemistry sections of Chemical Abstracts. Obviously, the impact differs considerably among the sections under the biochemistry heading. The CPP indicator illustrates that citation habits vary extensively not only between fields but also within fields. For the biochemistry sections of Chemical Abstracts the average citation rate varies between 2.37 and 19.67 (see Table 2). This suggests that the classification scheme of Thomson Reuters may not provide sufficient resolution for assessing the impact of research groups, for example in the case of highly specialized fields of research with a small scientific community. In comparative analysis, the selection of inadequate reference standards may result in invalid conclusions and thus affect the fairness of research evaluation. Suppose we evaluate a research group that publishes research findings in nonmammalian biochemistry: We could apply (a) the reference standard for "biology \& biochemistry" (13.78) or "chemistry" (7.45) published in the Essential Science Indicators of Thomson Reuters, ${ }^{2}$ (b) the reference standard for "biochemistry \& molecular biology" (18.52) based on the subject categories of Thomson Reuters (see Table 1), or (c) the reference standard for "nonmammalian biochemistry" (10.90) based on the sections of Chemical Abstracts (see Table 2). The different values of the reference standard show that the evaluation of research performance is highly dependent on the reference standard chosen. Depending on the frame of reference selected in the above example, the values of the reference standard vary between 7.45 ("chemistry") and 18.52 ("biochemistry \& molecular biology").

${ }^{2}$ The reference standards for papers published in 2000 were retrieved January 1, 2005 from the Essential Science Indicators. The analysis covered the time period January 1, 1994 to October 10, 2004. 


\section{Discussion}

The reference standards based on the fields of the Essential Science Indicators, the subject categories of Thomson Reuters, and the sections of Chemical Abstracts not only reflect different resolutions of fields but also are based on different data. When comparing, it is important where possible to consult reference standards that are based on the same data as are the citation rates of the institute or research group. Furthermore, reference standards should take into account the document type (research article, letter, note, review) and the publication year of papers [KOSTOFF \& AL., 2005; VAN LEEUWEN \& AL., 2003; VAN RAAN, 2004].

As a prerequisite to valid reference standards based on the sections of Chemical Abstracts, we must assume that the subject classification of Chemical Abstracts is not affected by what is called the "indexer effect." That is, we must assume that indexers assign the relevant main section to papers. According to BRAAM \& BRUIL [1992], the indexing of Chemical Abstracts in 80 main sections is in accordance with author classification preferences for $80 \%$ of papers. Despite the quality of the indexing, we should be aware of the indexing policies behind Chemical Abstracts, which may change over time, in order to prevent invalid conclusions.

The downside of subject classification schemes as well as journal classification schemes is their inflexible and delayed nature. In order to track scientific advancement and to represent emerging areas of research, it is essential that the classification scheme is continually revised and updated. Furthermore, classification schemes usually fail to represent research paradigms or research fronts. It is, however, reasonable to assume that the probability of being cited differ among research paradigms or research fronts, respectively.

A limitation of Chemical Abstracts concerns the institutional affiliation of contributing authors. In contrast to the Science Citation Index of Thomson Reuters, Chemical Abstracts provides only one address per paper, mainly the institutional affiliation of the reprint author. Chemical Abstracts is thus primarily suitable for citation analysis on the basis of publication lists compiled or verified by the researchers themselves, the so-called bottom-up approach (cf. [VAN LEEUWEN, 2007]). For the topdown approach, which identifies the relevant publications of a nation, an institution or a research group on the basis of the address information available in the bibliographic database, Chemical Abstracts is an inappropriate starting point in assessing research performance.

Nevertheless, the sections of Chemical Abstracts seem to be a promising basis for reference standards in chemistry and related fields for four reasons: (1) The wider coverage of the pertinent literature, (2) the quality of indexing, (3) the assignment of papers published in multidisciplinary and general journals to their respective fields, and (4) the resolution of fields on a lower level (e.g. mammalian biochemistry) than in 
journal classification schemes (e.g. biochemistry \& molecular biology). The proposed reference standard is transparent, reproducible and overcomes some limitations of the journal classification scheme of Thomson Reuters. For this analysis, we determined the reference standards for the biochemistry heading of Chemical Abstracts and their sections as an example. Determining the values of the reference standard in CAplus on STN International is a costly and time-consuming but feasible task. In principle, it is possible to even refine fields by (a) using subsection placement or (b) including index terms of Chemical Abstracts. The determination of reference standards for topics is beset, however, with some problems (e.g. the number of papers may be too small to determine a reliable reference standard).

The $\mathrm{CPP} / \mathrm{FCSm}_{\mathrm{CA}}$ indicator is designed to assess the research performance of universities, institutes and research groups in chemistry and related fields. It is intended to supplement rather than replace other relative indicators. In further analysis, we will examine the adequacy of the reference standard based on the sections of Chemical Abstracts for the evaluation of research groups.

The authors thank STN International for its generous financial support of online searching, Dr. Werner Marx at the Max Planck Institute for Solid State Research in Stuttgart (Germany) for assisting with citation analysis in CAplus, and Dr. András Schubert at the Information Science and Scientometrics Research Unit (ISSRU) in Budapest (Hungary) for providing the reference standards based on the subject categories of the Journal Citation Reports.

\section{References}

AKSNES, D. W. (2005), Citation rates and perceptions of scientific contribution, Journal of the American Society for Information Science and Technology, 57 : 169-185.

BRAAM, R. R., BRUIL, J. (1992), Quality of indexing information: Authors' views on indexing of their articles in Chemical Abstracts online CA-File, Journal of Information Science, 18 : 399-408.

Chemical ABSTRACTS Service (1997), Subject Coverage and Arrangement of Abstracts by Sections in Chemical Abstracts, Columbus.

Chemical Abstracts Service (1999), Chemical Abstracts. Content Description, Columbus.

Chemical Abstracts Service (2005), Cited References in Caplus and CA, STNotes, 24, available at: http://www.cas.org/support/stngen/stnotes/.

GlänZEL, W., Schubert, A. (2003), A new classification scheme of science fields and subfields designed for scientometric evaluation purposes, Scientometrics, $56: 357-367$.

Glänzel, W., Schubert, A., Czerwon, H. J. (1999), An item-by-item subject classification of papers published in multidisciplinary and general journals using reference analysis, Scientometrics, $44: 427-439$.

Hamilton, K. S. (2003), Subfield and Level Classification of Journals (CHI No. 2012-R), CHI Research, Haddon Heights.

KATZ, J. S., HiCKS, D. (1995), The classification of interdisciplinary journals: A new approach. In: M. E. D. Koenig, A. Bookstein (Eds), Proceedings of the Fifth Biennial Conference of the International Society for Scientometrics and Informetrics. Learned Information Inc., Medford, pp. 245-254.

Kostoff, R. N. (2002), Citation analysis of research performer quality, Scientometrics, 53 : 49-71. 
KOSTOFF, R. N., MARTINEZ, W. L. (2005), Is citation normalization realistic? Journal of Information Science, $31: 57-61$.

MARTIN, B. R., IRVINE, J. (1983), Assessing basic research - Some partial indicators of scientific progress in radio astronomy, Research Policy, $12: 61-90$.

MARX, W., SChIER, H., WANITSCheK, M. (2001), Citation analysis using online databases: Feasibilities and shortcomings, Scientometrics, $52: 59-82$.

Moed, H. F. (2005), Citation Analysis in Research Evaluation, Springer, Berlin.

NeUHAUS, C., DANIEL, H.-D. (2008), Data sources for performing citation analysis - An overview, Journal of Documentation, 64 : 193-210.

Noma, E. (1986), Subject Classification and Influence Weights for 3000 Journals (CHI No. 8602F), CHI Research, Cherry Hill.

RIDLEY, D. D. (2001), Citation searches in on-line databases: Possibilities and pitfalls, Trends in Analytical Chemistry, $20: 1-10$.

Rinia, E. J., De Lange, C., Moed, H. F. (1993), Measuring national output in physics: Delimitation problems, Scientometrics, $28: 89-110$.

SChUBERT, A., BRAUn, T. (1996), Cross-field normalization of scientometric indicators, Scientometrics, $36: 311-324$.

VAN LEEUWEN, T. N. (2007), Modelling of bibliometric approaches and importance of output verification in research performance assessment, Research Evaluation, 16 : 93-105.

Van Leeuwen, T. N., Visser, M. S., Moed, H. F., Nederhof, T. J., Van RaAn, A. F. J. (2003), The Holy Grail of science policy: Exploring and combining bibliometric tools in search of scientific excellence, Scientometrics, $57: 257-280$.

VAn RaAn, A. F. J. (2004), Measuring science. In: H. F. Moed, W. GlänZel, U. Schmoch (Eds), Handbook of Quantitative Science and Technology Research. The Use of Publication and Patent Statistics in Studies of S\&T Systems, Kluwer, Dordrecht, pp. 19-50.

VINKLER, P. (1986), Evaluation of some methods for the relative assessment of scientific publications, Scientometrics, $10: 157-177$.

WhitLEy, K. M. (2002), Analysis of SciFinder Scholar and Web of Science citation searches, Journal of the American Society for Information Science and Technology, 53 : 1210-1215. 